\title{
Single versus double lung transplantation for fibrotic disease-systematic review
}

\author{
Ashley R. Wilson-Smith ${ }^{1,2 \#}$, Yong Sul Kim ${ }^{1 \#}$, Georgina E. Evans ${ }^{1}$, Tristan D. Yan ${ }^{2,3,4}$ \\ ${ }^{1}$ The University of New South Wales, Medicine, Sydney, Australia; ${ }^{2}$ The Collaborative Research Group (CORE), Macquarie University, Sydney, \\ Australia; ${ }^{3}$ The Royal Prince Alfred Hospital, Sydney, Australia; ${ }^{4}$ Sydney Adventist Hospital, University of Sydney, Sydney, Australia \\ \#These authors contributed equally to this work. \\ Correspondence to: Ashley R. Wilson-Smith. The Collaborative Research Group (CORE), Sydney, Australia. Email: wilsonsmithash597@gmail.com.
}

\begin{abstract}
Background: Lung transplantation has long been the accepted therapy for end-stage pulmonary fibrotic disease. Presently, there is an ongoing debate over whether single or bilateral transplantation is the most appropriate treatment for end-stage disease, with a paucity of high-quality evidence comparing the two approaches head-to-head.
\end{abstract}

Methods: This review was performed in accordance with PRISMA recommendations and guidance. Searches were performed on PubMed Central, Scopus and Medline from dates of database inception to September 2019. For the assessed papers, data was extracted from the reviewed text, tables and figures, by two independent authors. Estimated survival was analyzed using the Kaplan-Meier method for studies where time-to-event data was provided.

Results: Overall, 4,212 unique records were identified from the literature search. Following initial screening and the addition of reference list findings, 83 full-text articles were assessed for eligibility, of which 17 were included in the final analysis, with a total of 5,601 patients. Kaplan-Meier survival analysis illustrated improved survival in patients receiving bilateral lung transplantation (BLTx) than in those receiving unilateral transplantation for idiopathic pulmonary fibrosis at all time intervals, with aggregated survival for BLTx at 57\%, 35.3\% and 24\% at 5-, 10- and 15-year follow-up, respectively. Survival rates for SLTx were $50 \%, 27.8 \%$ and $13.9 \%$, respectively.

Conclusions: Whilst a number of studies present conflicting results with respect to short-term transplantation outcomes, BLTx confers improved long-term survival over SLTx, with large-scale registries supporting findings from single- and multi-center studies. Through an aggregation of published survival data, this meta-analysis identified improved survival in patients receiving BLTx versus SLTx at all time intervals.

Keywords: Lung transplantation; fibrotic disease; outcomes

Submitted Nov 16, 2019. Accepted for publication Dec 13, 2019.

doi: 10.21037/acs.2019.12.04

View this article at: http://dx.doi.org/10.21037/acs.2019.12.04

\section{Introduction}

Lung transplantation has long been an accepted therapy for end-stage pulmonary fibrotic disease given the poor long-term prognosis of patients managed with medical and conservative treatment (1). Presently, there is an ongoing debate over whether single (SLTx) or bilateral (BLTx) lung transplantation is the most appropriate treatment, with a paucity of high-quality evidence comparing the two approaches head-to-head (2). As such, practice is still largely specific to the institution or to the surgeon's preference. Historically, SLTx has been used under the rationale that it is a more limited operation and hence is more appropriate for high-risk candidates; however, as surgical techniques have improved, bilateral transplantationparticularly with its increasingly acceptable long-term 
morbidity and mortality—has become predominant. Given the heterogeneity of interstitial lung diseases, particularly in terms of their etiology and pathophysiology, substantive subgroup analysis to date has been hindered outside of registry findings, with patient outcomes highly variable within the literature (3). The present systematic review will detail the mid- and late-term outcomes after SLTx vs. BLTx for pulmonary fibrotic disease and will provide an aggregation of the present data on survival outcomes.

\section{Methods}

\section{Literature search}

This review was performed in accordance with PRISMA recommendations and guidance $(4,5)$. Electronic searches were performed on PubMed Central, Scopus and Medline from dates of database inception to September 2019, using the terms ("lung transplantation" OR "lung transplant") AND ("single" OR "unilateral" OR "bilateral" OR "double") AND ("pulmonary fibrosis" OR "idiopathic pulmonary fibrosis") as either keywords or MeSH headings. After removal of duplicate records, studies were screened according to their titles and abstracts, then reviewed according to the inclusion and exclusion criteria detailed below by two independent authors (AR Wilson-Smith, YS Kim). Conflicts were resolved by the senior researcher (AR Wilson-Smith). A PRISMA diagram of the search strategy is presented in Figure S1. Additional references for discussion were obtained by reference list searches, or via targeted database searches.

\section{Inclusion and exclusion criteria}

Studies were eligible for this review if they had at least ten patients in their cohorts and where transplantation (either SLTx or BLTx) was indicated primarily for idiopathic pulmonary fibrosis (IPF). Follow-up of a minimum of one year was also required. Non-English records, review articles, conference and paper abstracts, editorials, letters, case reports, series, and opinions were excluded. Studies were excluded from analysis if they failed to delineate between SLTx and BLTx outcomes, or if no mention was made of the outcome between the two approaches qualitatively.

Studies which aggregated etiologies in their survival analysis (e.g., IPF + cystic fibrosis + chronic obstructive pulmonary disease) were excluded from quantitative analysis, as were emergent surgeries. Duplicate studies were removed prior to the commencement of the literature screen and only the most up-to-date references from ongoing studies or registries were reviewed for statistical aggregation to minimize patient overlap. Studies were also excluded if they did not present baseline patient characteristics, or if the study was centered on pediatric patients (i.e., those $<18$ years).

\section{Data extraction, statistical analysis and presentation}

For the assessed papers, data was extracted from the reviewed text, tables and figures. Data was extracted independently by two independent authors (AR WilsonSmith, YS Kim) into Microsoft Excel. Discrepancies were reviewed and discussed until a consensus was reached. Findings are presented in Tables 1-3. Estimated survival was analyzed using the Kaplan-Meier method in studies where time-to-event data was provided (6). Censoring was assumed to be constant, unless the particular curve had a long follow-up of only minimal patients-in which case, censoring was manually entered. Death events and censoring data were compiled for the entire patient cohort and overall survival curves were produced using R Studio (7).

\section{Quality assessment of included studies}

An appraisal schema based on the Canadian National Institute of Health Economics' (CNIHE) Quality Assessment Tool (i.e., the modified Delphi technique) for case series studies was employed to evaluate all included studies (Figure S2). Studies were categorized based on the following domains: clarity of study objective, adequate description of the study population, description of the intervention, adequate reporting of outcome measures, appropriate reporting of results/conclusions (quality findings are listed in Table 1, criteria are provided in Figure S2). Studies were considered to be of high quality if they addressed at least 15 of the 19 criteria outlined in the CNIHE tool. Moderate quality was defined as $13-15$ of 19 and low quality below 13 of 19 .

\section{Results}

Overall, 4,212 unique records were identified from the literature search (Figures S1,S3). Following initial screening and the addition of reference list findings, 83 full-text articles were assessed for their eligibility, of which 17 were included in the final analysis. Six studies were included 


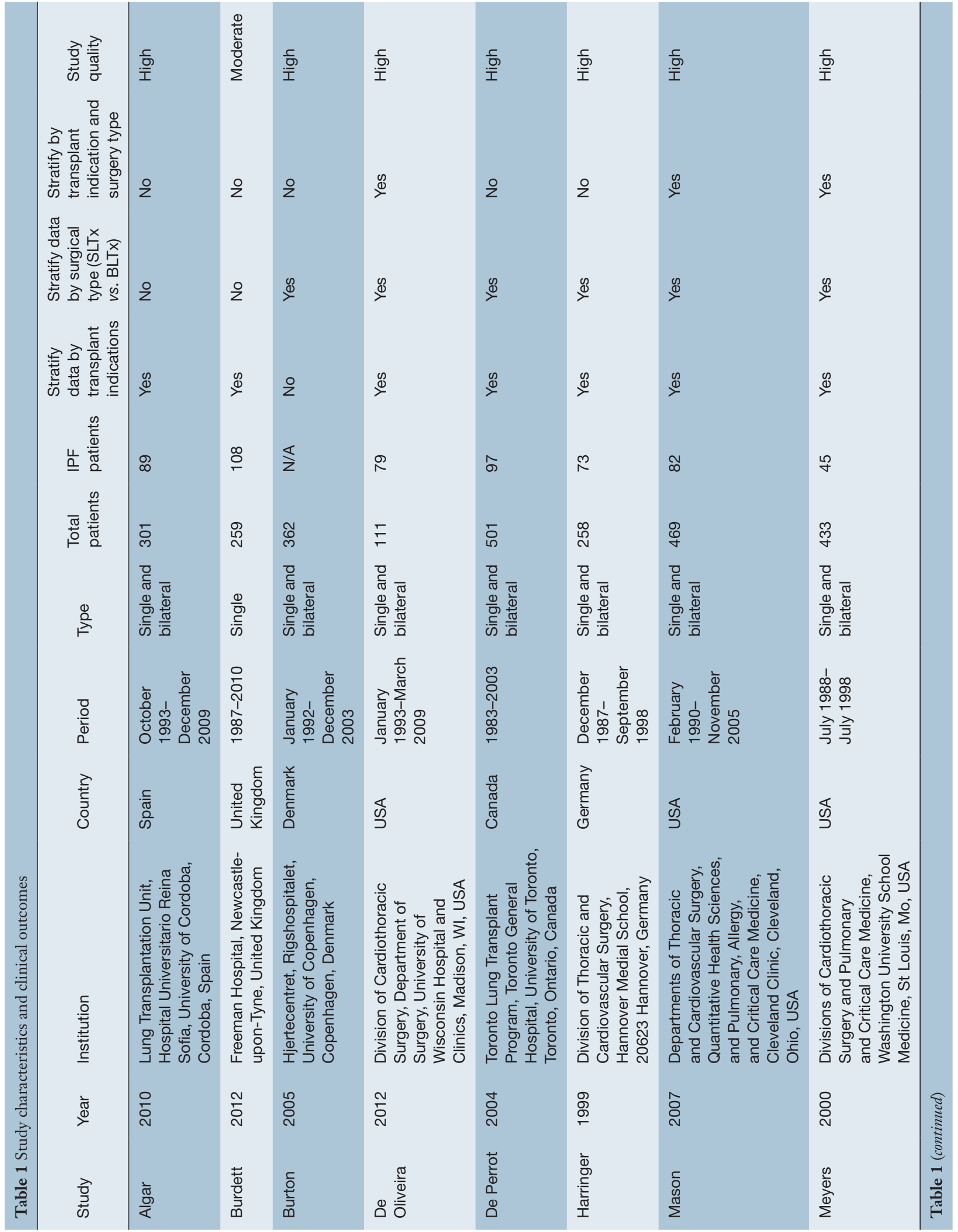




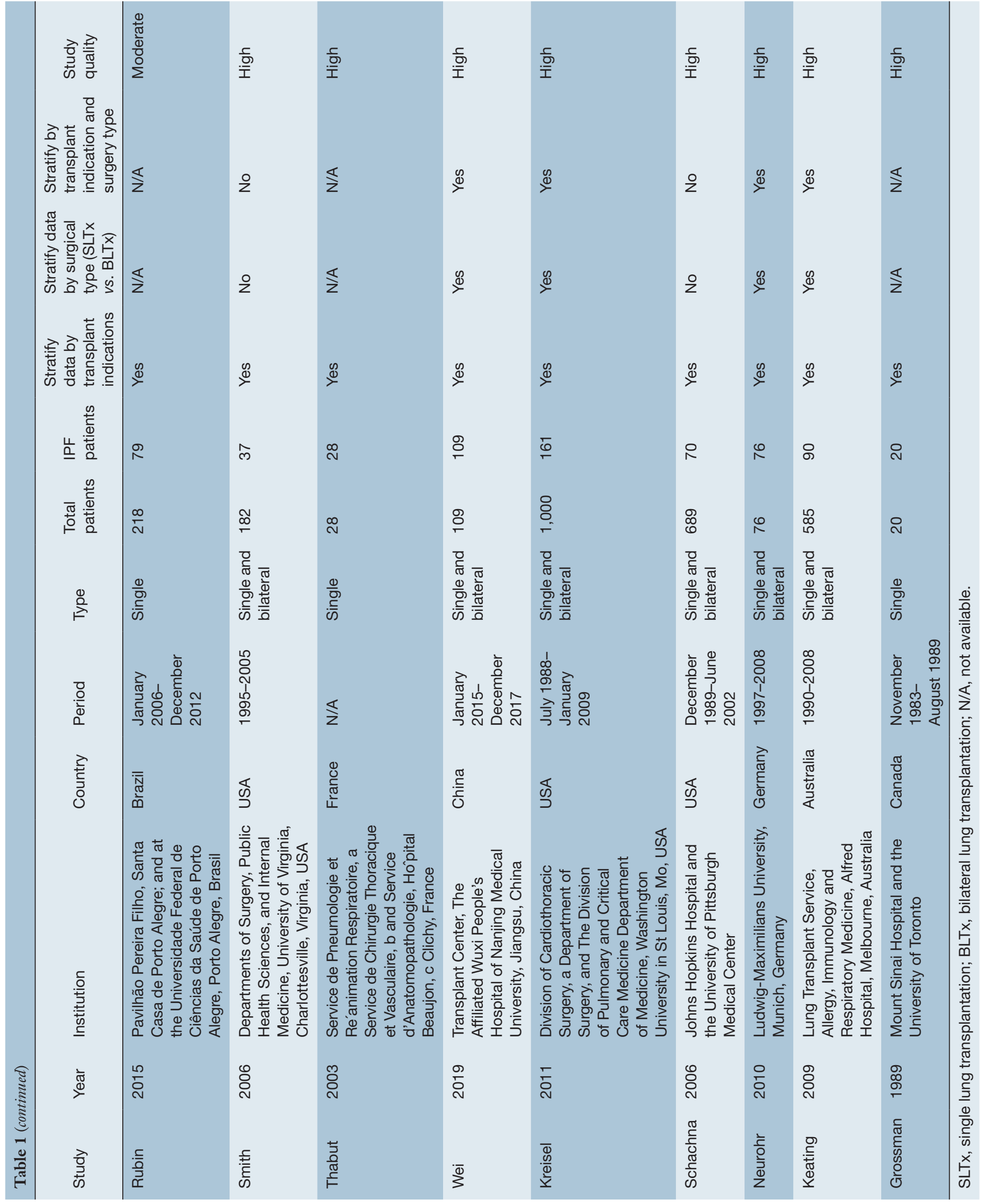




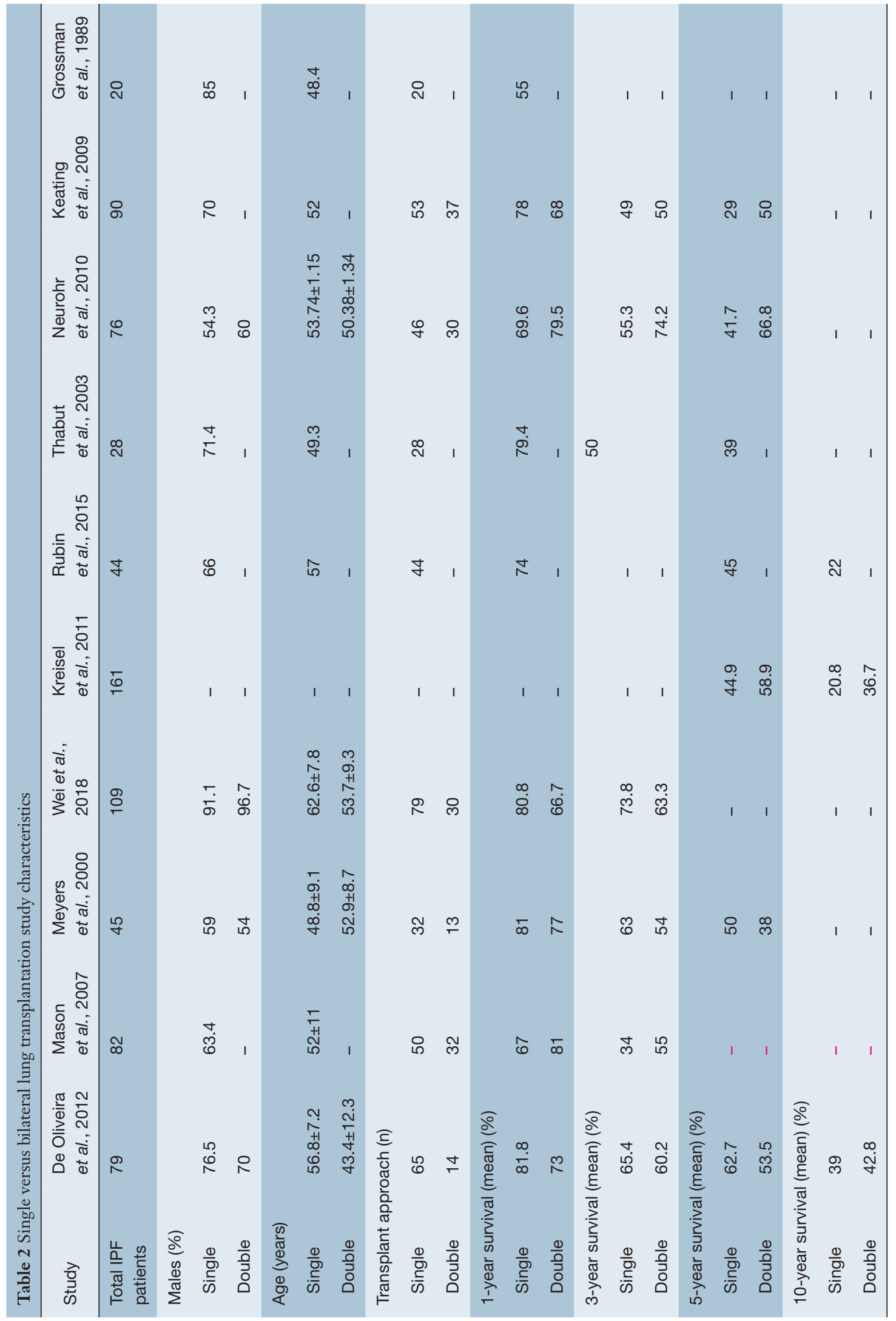




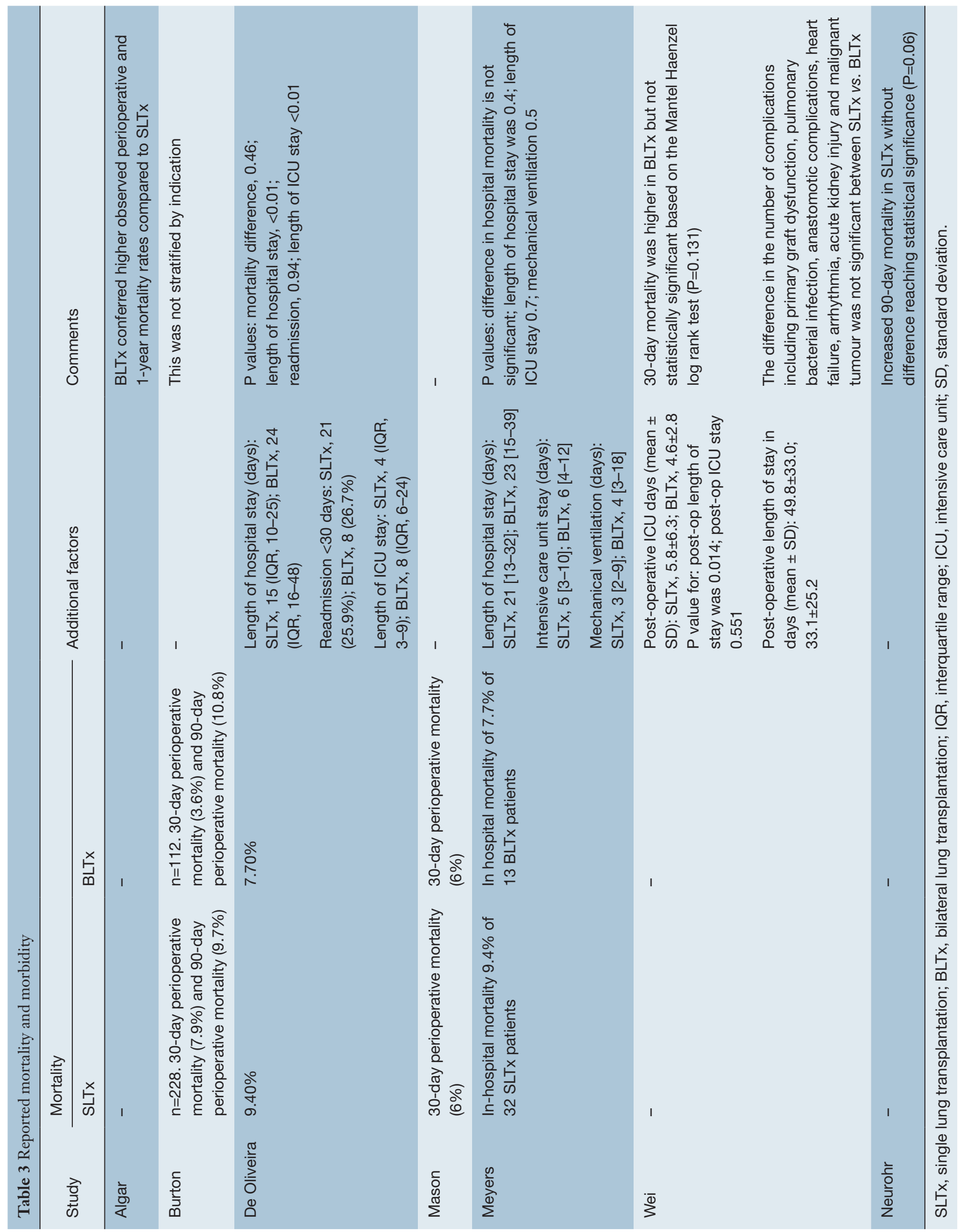




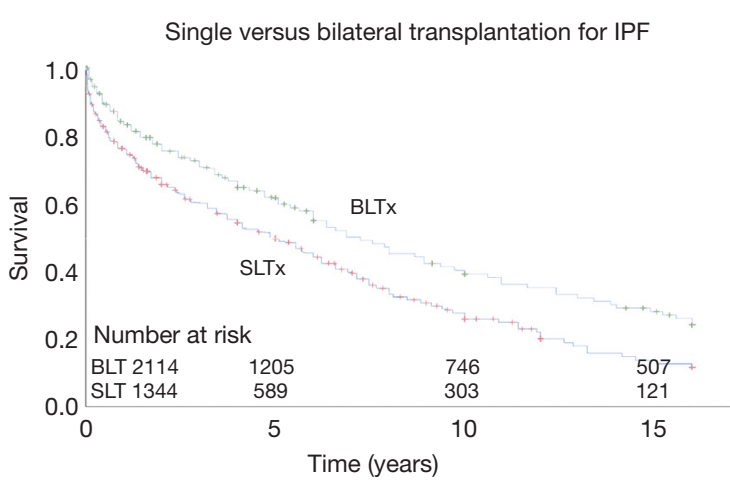

Figure 1 Kaplan-Meier survival analysis for single versus bilateral lung transplantation in IPF. IPF, idiopathic pulmonary fibrosis; BLTx, bilateral lung transplantation; SLTx, single lung transplantation.

in the quantitative synthesis (8-13). The search of 4,212 records yielded 17 studies which met inclusion criteria, with a total of 5,601 patients. The majority of studies (90\%) were found to be of high quality according to the CNIHE tool, with the remainder as moderate quality. The majority of studies included in the final analysis were from European or United States centers, with the remainder being drawn from Asian, Canadian or Central American centers.

\section{Survival-SLTx vs. BLTx}

All curves digitized and aggregated in this study were unadjusted. Aggregated survival for SLTx at 5-, 10and 15 -year time intervals was $50 \%, 27.8 \%$ and $13.9 \%$, respectively. Survival for BLTx was $57 \%, 35.3 \%$ and $24 \%$, respectively (Figure 1).

\section{Morbidity-SLTx vs. BLTx}

Reported morbidity findings are detailed in Table 3. Three studies outlined morbidity data quantitatively $(8,10,11)$. Length of hospital stay was statistically significant in two studies in favor of SLTx. Length of ICU stay was only significant in one study in favor of SLTx. All other reported complication rates were not statistically significant between the two surgical modalities.

\section{Discussion}

\section{SLTx vs. BLTx: head-to-head studies}

Out of the seven studies that present survival rates of BLTx vs. SLTx specifically for IPF patients, three of these studies establish improved survival in patients receiving SLTx. De Oliveira et al. demonstrate in 79 patients that survival is better in SLTx up to 5 years. The 1-, 3-, 5-year survival rates of SLTx vs. BLTx were $81.8 \%$ vs. $73 \%, 65.4 \%$ vs. $60.2 \%, 62.7 \%$ vs. $53.5 \%(\mathrm{P}=0.68)$, respectively (8). The 10 -year survival rate was $39 \%$ vs. $42.8 \%$, respectively. Meyers et al. demonstrate survival rates of SLTx vs. BLTx being $81 \%$ vs. $77 \%, 63 \%$ vs. $54 \%$ and $50 \%$ vs. $38 \%$ for the 1-, 3- and 5-year endpoints $(\mathrm{P}=0.42)(10)$. These rates were established in 32 SLTx and 13 sequential BLTx; no difference in in-hospital mortality between SLTx (9.40\%) and BLTx $(7.70 \%)$ was noted.

The most recent single center study by Wei et al. (11) demonstrated survival rates in a cohort of 109 patients of $80.8 \%$ vs. $66.7 \%$ and $73.8 \%$ vs. $63.3 \%$ for SLTx vs. BLTx, at 1 - and 2-year survival, respectively $(\mathrm{P}=0.13)$. When the survival rates of SLTx $v s$. BLTx in IPF patients are further stratified by age $<60$ and age $>60$, SLTx had significantly greater survival in the over-60 age-group $(\mathrm{P}=0.008)$.

The other studies align with the findings of the UNOS Registry and ISHLT registry, which outline that BLTx has improved survival for IPF patients over SLTx (14-22). In their cohort of 469 patients, 82 of which had IPF, Mason et al. found that patients receiving BLTx had better riskunadjusted survival than those receiving SLTx, with 1-year survival at $81 \%$ and $67 \%$, and 5 -year survival at $55 \%$ and $34 \%$, respectively. BLTx survival rates were similar to those for non-IPF indications (9). Following matched analysis, the benefit of BLTx over SLTx was not maintained, with perioperative mortality and short-term postoperative mortality both at $6 \%(10)$.

Neurohr et al. found a significant survival benefit of BLTx over SLTx in their cohort of 76 patients at 1-year and in overall survival $(\mathrm{P}=0.026)(13)$. Although SLTx had a higher percentage of acute rejection (35.6\%) compared to BLTx (29.6\%), this was not statistically significant; however, there was a significantly higher number of deaths in SLTx compared to BLTx as a result of the development of bronchiolitis obliterans. Kreisel et al. demonstrate a higher survival rate in BLTx for the 5- and 10-year mark (23). Interestingly, Kreisel extracted data from 1988 to 2009 , including the data used in the Meyers et al. (10) study from the same institution (1988-1998); however, it appears that the data from the extended 10-year followup reversed the survival benefit that SLTx had over BLTx found by Meyers et al. (10) One reason for this finding may be improved surgical technique of BLTx within the last ten 
years, or alternatively, BLTx may in fact have improved long-term survival benefits after the initial convalescence period. Keating et al. (12) demonstrated a survival rate pattern similar to that of De Oliveira et al. (8) up to the 3-year mark, the survival rate was higher for SLTx compared to BLTx; however, at the 5-year mark, BLTx conferred a higher survival rate of $50 \%$ compared to SLTx at $29 \%$.

Six studies outlined findings for IPF patients undergoing transplantation but did not stratify for surgical approach with quantitative analysis. For instance, Algar et al. found that survival did not differ between SLTx and BLTx at long-term follow-up, noting that whilst BLTx conferred increased mortality in the short-term, the long-term survival benefit offset the initial mortality (24). A number of studies compared SLTx and BLTx, but did not differentiate for etiology. de Perrot et al. (15) found in their cohort of 501 patients, long-term survival was higher in patients receiving BLTx vs. patients receiving SLTx $(\mathrm{P}=0.07)$; when separately analyzing for etiology (i.e., cystic fibrosis, chronic obstructive pulmonary disease, IPF, etc.) this effect disappeared; however, no data were presented in-text.

\section{Database analysis of IPF patient outcomes}

Villavicencio et al. (25) presents the most recent data comparing survival outcomes of SLTx vs. BLTx in IPF recipients. This study includes 9,191 lung transplant recipients with IPF from 1987 to 2015. They have demonstrated that BLTx has improved survival outcomes in IPF patients compared to SLTx across all lung allocation scores and additionally when adjusted for age, excepting those exceeding 70 years $(\mathrm{P}<0.001)$. The International Society for Heart and Lung Transplantation Registry (ISHLT) have collated the survival outcomes of SLTx $v s$. BLTx in IPF patients and presented their data on transplant recipients with IPF from January 1990 to June 2016. Kaplan-Meier curves were produced from 1,043 SLTx patients with IPF and 1,936 BLTx patients with IPF. BLTx was shown to have improved survival outcomes compared to SLTx at each year from the $1^{\text {st }}$ year to the $16^{\text {th }}$ year $(\mathrm{P}<0.0001)$.

\section{Limitations}

To the authors' knowledge, this is the most recent and thorough systematic review of the literature comparing SLTx to BLTx in the context of IPF and the only review to incorporate an aggregation of survival data across singleand multi-center studies and registries.

However, are several limitations with respect to the present review. The number of single- and multi-center studies analyzing the survival outcomes of IPF patients with clear stratification of which patients received SLTx and BLTx was low, with only 17 studies meeting the final inclusion criteria. The majority of these studies provided survival outcomes of IPF recipients compared to other transplant recipients of varying indications, and/or survival outcomes comparing SLTx vs. BLTx in all transplant recipients. Only six studies—with one potentially replicated dataset-were able to stratify survival outcomes by SLTx $v s$. BLTx specifically for IPF patients.

The limited number of studies with survival outcomes and stratification between surgical approaches prevented meaningful quantitative analysis of the single- and multicenter studies, requiring an aggregation of the findings from large registries. It is likely that there is a degree of patient overlap, as patients within the registries would have been drawn from these single- and multi-center studies; however, using the methods of Sampson et al. who present techniques for identifying cohort overlap, this was mitigated as far as possible (26). Although registry studies are extremely useful (i.e., they benefit from large numbers that allow for sound statistical analysis), it is of note that they include and collate data from a wide array of centers, with surgeons of variable expertise, using different surgical protocols and incomplete patient data. Hence, in centers which have always preferred one technique over another become highly proficient in that technique; it would not be surprising to note outcomes that go against the findings from registry data.

The primary constraint of this review is that the single- and multi-center studies identified have often yielded conflicting results, with largely uninterpretable or sparsely reported data (from a meta-analytical perspective) supporting their recommendations; often, no differentiation is made between the indications for transplantation (e.g., cystic fibrosis, IPF, etc.) or which patients are receiving what type of intervention (i.e., SLTx or BLTx). Further, very limited morbidity data were reported, with only three studies comparing SLTx and BLTx for IPF providing quantitative results (Table 3). Often, authors would make comments in their discussions noting that one technique was more favorable in terms of survival or complications, but with no analysis to substantiate the claims.

In order to carry out definitive analysis, it is critical that patients with different pathologies are not grouped and 
vice versa for transplantation technique, as the level of heterogeneity strongly calls into question the validity of the findings. It is imperative that for future analysis of survival outcomes, clinicians and researchers provide datasets which have been stratified appropriately for etiology as well as for surgical approach.

\section{Conclusions}

Whilst a number of studies present conflicting results with respect to short-term transplantation outcomes, the consensus is that BLTx confers improved long-term survival over that of SLTx, with large-scale registries supporting the findings from single- and multi-center studies. Through an aggregation of the present survival data, this meta-analysis identified improved survival in patients receiving BLTx versus those receiving SLTx at all time intervals.

\section{Acknowledgments}

None.

\section{Footnote}

Conflicts of Interest: The authors have no conflicts of interest to declare.

\section{References}

1. Schaffer JM, Singh SK, Reitz BA, et al. Single- vs doublelung transplantation in patients with chronic obstructive pulmonary disease and idiopathic pulmonary fibrosis since the implementation of lung allocation based on medical need. JAMA 2015;313:936-48.

2. Puri V, Patterson GA, Meyers BF. Single versus bilateral lung transplantation: do guidelines exist? Thorac Surg Clin 2015;25:47-54.

3. Kistler KD, Nalysnyk L, Rotella P, et al. Lung transplantation in idiopathic pulmonary fibrosis: a systematic review of the literature. BMC Pulm Med 2014;14:139.

4. Phan K, Tian DH, Cao C, et al. Systematic review and meta-analysis: techniques and a guide for the academic surgeon. Ann Cardiothorac Surg 2015;4:112-22.

5. Liberati A, Altman DG, Tetzlaff J, et al. The PRISMA statement for reporting systematic reviews and metaanalyses of studies that evaluate healthcare interventions: explanation and elaboration. BMJ 2009;339:b2700.
6. Guyot P, Ades AE, Ouwens MJ, et al. Enhanced secondary analysis of survival data: reconstructing the data from published Kaplan-Meier survival curves. BMC Med Res Methodol 2012;12:9.

7. Crowther MJ, Riley RD, Staessen JA, et al. Individual patient data meta-analysis of survival data using Poisson regression models. BMC Med Res Methodol 2012;12:34.

8. De Oliveira NC, Osaki S, Maloney J, et al. Lung transplant for interstitial lung disease: outcomes for single versus bilateral lung transplantation. Interact Cardiovasc Thorac Surg 2012;14:263-7.

9. Mason DP, Brizzio ME, Alster JM, et al. Lung transplantation for idiopathic pulmonary fibrosis. Ann Thorac Surg 2007;84:1121-8.

10. Meyers BF, Lynch JP, Trulock EP, et al. Single versus bilateral lung transplantation for idiopathic pulmonary fibrosis: a ten-year institutional experience. J Thorac Cardiovasc Surg 2000;120:99-107.

11. Wei D, Gao F, Wu B, et al. Single versus bilateral lung transplantation for idiopathic pulmonary fibrosis. Clin Respir J 2019;13:376-83.

12. Keating D, Levvey B, Kotsimbos T, et al. Lung transplantation in pulmonary fibrosis: challenging early outcomes counterbalanced by surprisingly good outcomes beyond 15 years. Transplant Proc 2009;41:289-91.

13. Neurohr C, Huppmann P, Thum D, et al. Potential functional and survival benefit of double over single lung transplantation for selected patients with idiopathic pulmonary fibrosis. Transpl Int 2010;23:887-96.

14. Burdett C, Butt T, Lordan J, et al. Comparison of single lung transplant with and without the use of cardiopulmonary bypass. Interact Cardiovasc Thorac Surg 2012;15:432-6; discussion 436.

15. de Perrot M, Chaparro C, McRae K, et al. Twenty-year experience of lung transplantation at a single center: Influence of recipient diagnosis on long-term survival. J Thorac Cardiovasc Surg 2004;127:1493-501.

16. Harringer W, Wiebe K, Strüber M, et al. Lung transplantation--10-year experience. Eur J Cardiothorac Surg 1999;16:546-54.

17. Rubin AS, Nascimento DZ, Sanchez L, et al. Functional improvement in patients with idiopathic pulmonary fibrosis undergoing single lung transplantation. J Bras Pneumol 2015;41:299-304.

18. Smith PW, Wang H, Parini V, et al. Lung transplantation in patients 60 years and older: results, complications, and outcomes. Ann Thorac Surg 2006;82:1835-41; discussion 1841. 
19. Thabut G, Mal H. Outcomes after lung transplantation. J Thorac Dis 2017;9:2684-91.

20. Schachna L, Medsger TA Jr, Dauber JH, et al. Lung transplantation in scleroderma compared with idiopathic pulmonary fibrosis and idiopathic pulmonary arterial hypertension. Arthritis Rheum 2006;54:3954-61.

21. Grossman RF, Cooper JD. Lung transplantation. Immunol Allergy Clin North Am 1989;9:153-63.

22. Burton CM, Milman N, Carlsen J, et al. The Copenhagen National Lung Transplant Group: survival after single lung, double lung, and heart-lung transplantation. J Heart Lung Transplant 2005;24:1834-43.

23. Kreisel D, Krupnick AS, Puri V, et al. Short- and long-

Cite this article as: Wilson-Smith AR, Kim YS, Evans GE, Yan TD. Single versus double lung transplantation for fibrotic disease-systematic review. Ann Cardiothorac Surg 2020;9(1):10-19. doi: 10.21037/acs.2019.12.04 term outcomes of 1000 adult lung transplant recipients at a single center. J Thorac Cardiovasc Surg 2011;141:215-22.

24. Algar FJ, Espinosa D, Moreno P, et al. Results of lung transplantation in idiopathic pulmonary fibrosis patients. Transplant Proc 2010;42:3211-3.

25. Villavicencio MA, Axtell AL, Osho A, et al. Single- Versus Double-Lung Transplantation in Pulmonary Fibrosis: Impact of Age and Pulmonary Hypertension. Ann Thorac Surg 2018;106:856-63.

26. Sampson M, McGowan J, Cogo E, et al. Managing database overlap in systematic reviews using Batch Citation Matcher: case studies using Scopus. J Med Libr Assoc 2006;94:461-3, e219. 

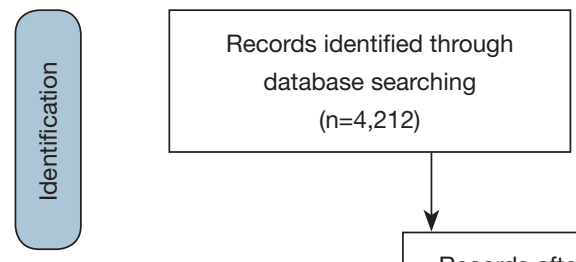

Additional records identified through reference list searches $(n=4,212)$

$$
(n=0)
$$
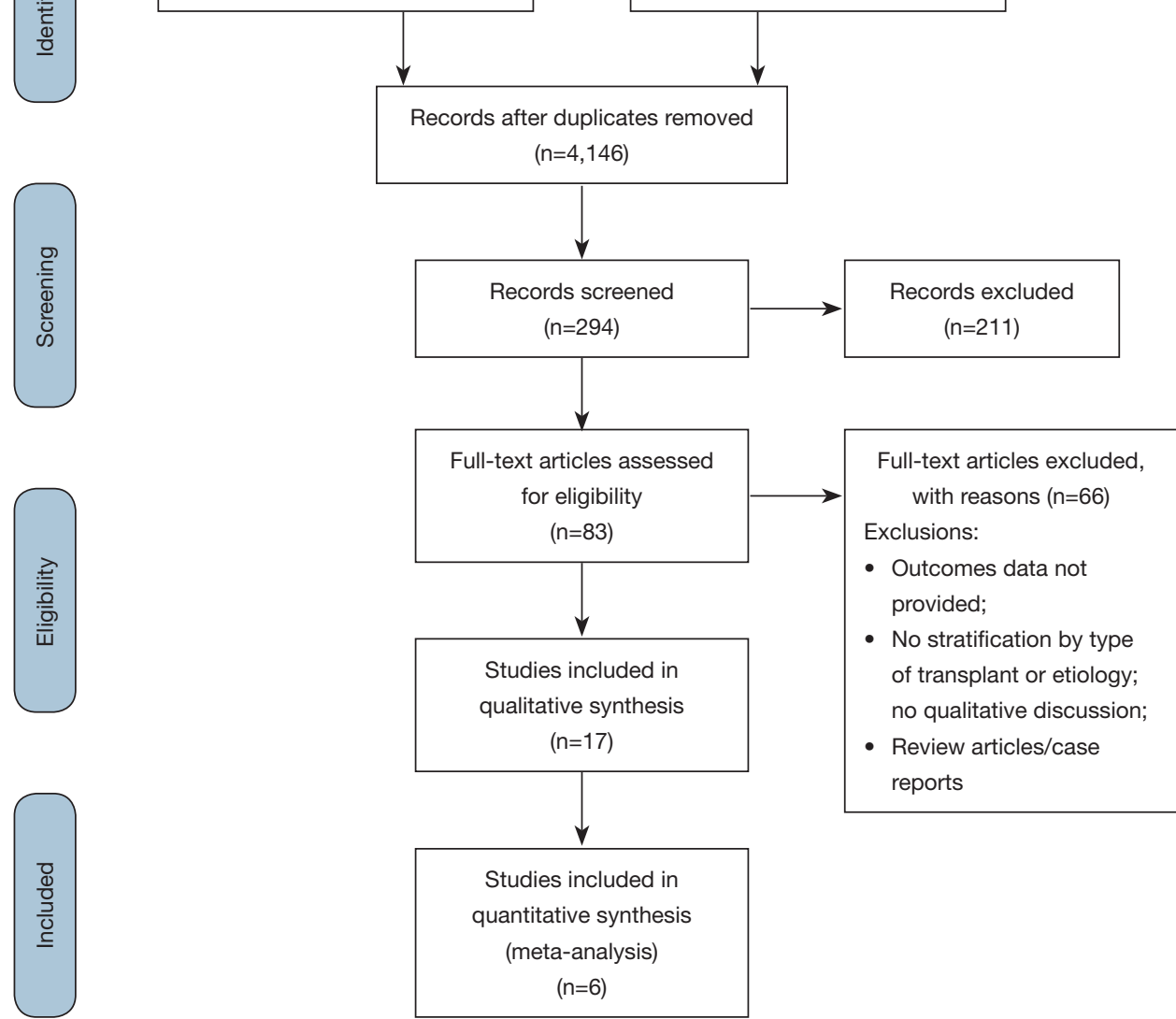

Studies included in quantitative synthesis (meta-analysis) $(n=6)$

Figure S1 PRISMA flow chart detailing the literature search process for mid- to late-term outcomes of single vs. bilateral lung transplantation in the setting of pulmonary fibrosis. 


\begin{tabular}{|c|c|}
\hline Criteria No. & Criterion definition \\
\hline 1 & Is the hypothesis/aim/objective of the study stated in the abstract, introduction, or methods section? \\
\hline 2 & Are the characteristics of the patients included in the study clearly described? \\
\hline 3 & Were the cases collected in more than one center? \\
\hline 4 & Are the eligibility criteria (inclusion and exclusion criteria) explicit and appropriate? \\
\hline 5 & Were patients recruited consecutively? \\
\hline 6 & Did patients enter the study at a similar point in the disease? \\
\hline 7 & Did the authors describe the intervention? \\
\hline 8 & In addition to intervention, did the patients receive any co-interventions? \\
\hline 9 & Was loss to follow-up reported? \\
\hline 10 & Are outcomes (primary, secondary) clearly defined in the introduction or methodology section? \\
\hline 11 & Did the authors use accurate (standard, valid, reliable) objective methods to measure the outcomes? \\
\hline 12 & Were outcomes assessed before and after intervention? \\
\hline 13 & Was the length of follow-up clearly described/reported? \\
\hline 14 & Were the statistical tests used to assess the primary outcomes appropriate? \\
\hline 15 & $\begin{array}{l}\text { Does the study provide estimates of the random variability in the data for the primary outcomes (e.g., } \\
\text { standard error, standard deviation, confidence intervals)? }\end{array}$ \\
\hline 16 & Was the analysis of outcomes based on intention to treat? \\
\hline 17 & Are adverse events that may be a consequence of the intervention reported? \\
\hline 18 & Are the conclusions of the study supported by results? \\
\hline 19 & $\begin{array}{l}\text { Is there a competing interest statement about the type and source of support received for the study or } \\
\text { about the relationship of the author(s) or other contributors with the manufacturer of the technology? }\end{array}$ \\
\hline
\end{tabular}

Figure S2 Quality appraisal criteria.

\begin{tabular}{|c|c|c|}
\hline Database & Search terms & Results \\
\hline $\begin{array}{c}\text { PubMed } \\
\text { Central }\end{array}$ & $\begin{array}{c}\text { ("lung transplantation" OR "lung transplant”) AND } \\
\text { ("single" OR "unilateral" OR "bilateral” OR "double") }\end{array}$ & 556 \\
& $\begin{array}{c}\text { AND ("pulmonary fibrosis" OR "idiopathic pulmonary } \\
\text { fibrosis") }\end{array}$ & \\
\hline
\end{tabular}

Figure S3 Search strategy supplementary. 